\title{
Aneurysm wall enhancement on magnetic resonance imaging as a risk factor for progression of unruptured vertebrobasilar dissecting aneurysms after reconstructive endovascular treatment
}

\author{
Yisen Zhang, MD, ${ }^{1}$ Binbin Sui, MD, ${ }^{2}$ Jian Liu, MD, ${ }^{1}$ Yang Wang, MD, ${ }^{3}$ Zhongbin Tian, MD, ${ }^{1}$ \\ Junfan Chen, MS, ${ }^{1}$ Zhongxue Wu, MD, ${ }^{1}$ and Xinjian Yang, MD, PhD ${ }^{1}$
}

'Department of Interventional Neuroradiology, Beijing Neurosurgical Institute and Beijing Tiantan Hospital, Capital Medical University, Beijing; ${ }^{2}$ Department of Neuroimaging, Beijing Tiantan Hospital, Capital Medical University, Beijing; and ${ }^{3}$ Department of Neurosurgery, the First Affiliated Hospital, Nanchang University, Nanchang, Jiangxi Province, China

OBJECTIVE The recurrence rate of vertebrobasilar dissecting aneurysms (VBDAs) after reconstructive endovascular treatment (EVT) is relatively high. The aneurysm wall enhancement on high-resolution MRI (HRMRI) reportedly predicts an unsteady state of an intracranial aneurysm. The authors used HRMRI to investigate the relationship between wall enhancement on HRMRI and progression of VBDAs after reconstructive EVT.

METHODS From January 2012 to December 2015, patients with an unruptured VBDA who underwent reconstructive EVT were enrolled in this study. Preoperative enhanced HRMRI was performed to evaluate radiological characteristics. The relationships between aneurysm wall enhancement and various potential risk factors were statistically analyzed. Follow-up angiographic examination was performed with digital subtraction angiography and conventional HRMRI. Cox regression analysis was performed to identify predictors of VBDA progression after reconstructive EVT.

RESULTS Eighty-two patients (12 women and 70 men, mean age $53.48 \pm 9.23$ years) with 83 VBDAs were evaluated in the current study. The average maximum diameter of the VBDAs was $11.30 \pm 7.90 \mathrm{~mm}$. Wall enhancement occurred in 43 VBDAs (51.81\%). Among all 83 VBDAs, 62 (74.70\%) were treated by stent-assisted coil embolization and $21(25.30 \%)$ by stenting alone. The mean duration of imaging follow-up among all 82 patients was 10.55 months (range 6-45 months), and 15 aneurysms (18.07\%) exhibited progression. The statistical analysis indicated no significant differences in age, sex, risk factors (high blood pressure, smoking, diabetes mellitus, and a high cholesterol level), VBDA stage, or VBDA size between enhanced and unenhanced VBDAs. Univariate Cox regression analysis showed that both the maximum diameter of the VBDAs and wall enhancement were associated with recurrence $(p<0.05)$. Multivariate Cox proportional hazard regression analysis showed that the maximum diameter of the VBDAs and wall enhancement on HRMRI were independent risk factors for aneurysm progression $(p<0.05)$.

CONCLUSIONS Aneurysm size and wall enhancement on HRMRI can predict the progression of VBDAs after reconstructive EVT.

https://thejns.org/doi/abs/10.3171/2016.11.JNS162433

KEY WORDS wall enhancement; high-resolution magnetic resonance imaging; intracranial dissecting aneurysm; endovascular treatment; progression; stent; vascular disorders

$\mathrm{S}$ PONTANEOUS intracranial dissecting aneurysms are rare. ${ }^{2}$ However, East Asian populations demonstrate a higher incidence of these types of aneurysms. ${ }^{14}$ The incidence rate may have increased due to the develop- ment of diagnostic technology. ${ }^{13}$ Intracranial vertebrobasilar dissecting aneurysms (VBDAs) are the most common type of intracranial dissecting aneurysms. ${ }^{14}$ Endovascular treatment (EVT) is now the most common operative meth-

ABBREVIATIONS 3D-TOF = 3D time of flight; $\mathrm{Cl}=$ confidence interval; DSA = digital subtraction angiography; EVT = endovascular treatment; FSE = fast spin-echo; HRMRI = high-resolution MRI; IMH = intramural hematoma; MRA = MR angiography; OR = odds ratio; TSE = turbo spin-echo; VBDA = vertebrobasilar dissecting aneurysm. SUBMITTED September 20, 2016. ACCEPTED November 21, 2016. 
od for treating VBDAs. ${ }^{11}$ Reconstructive EVT, an important type of EVT, still faces some challenges such as a relatively high recurrence rate. ${ }^{12}$ Identifying risk factors that may lead to VBDA progression after reconstructive EVT and taking appropriate measures to improve the efficacy of reconstructive EVT have become important issues.

High-resolution MRI (HRMRI) has been widely used to diagnose VBDAs. ${ }^{4}, 15$ Wall enhancement of a saccular aneurysm on HRMRI is a proven sign of inflammatory change and might predict an unsteady state of an intracranial saccular aneurysm. ${ }^{5,9}$ However, few studies have focused on wall enhancement of VBDAs. We hypothesized that wall enhancement might also predict an unsteady state of a VBDA as well as its progression after reconstructive EVT. In this study we used 3-T black-blood HRMRI to investigate the relationship between VBDA wall enhancement and aneurysm progression after reconstructive EVT, and we performed multivariate Cox proportional hazard regression analysis to identify risk factors that may lead to VBDA progression after reconstructive EVT.

\section{Methods \\ Study Population}

After obtaining IRB approval, we prospectively enrolled consecutive patients with unruptured VBDAs from January 2012 to December 2015. Digital subtraction angiography (DSA), MR angiography (MRA), and MRI were used to diagnose the intracranial dissecting aneurysms. An intracranial dissecting aneurysm was diagnosed when MRA or DSA showed fusiform dilation of vessels or the pearl-and-string sign or when MRI showed intimal flaps, the double-lumen sign, or an intramural hematoma (IMH). Patients planning to undergo reconstructive EVT underwent enhanced HRMRI examinations before EVT if they had no contraindication for enhanced HRMRI. The inclusion criteria were adult patients (age 18-80 years), our expectation of adequate patient safety during the examination, patients' willingness to participate in the study, and patients' ability to cooperate during the MRI examination. The exclusion criteria were the absence of radiological follow-up and a history of deconstructive EVT. Informed consent was obtained from each patient prior to the MRI examination. The VBDA stages were classified as acute ( $0-3$ days), early subacute (3-10 days), late subacute (10-60 days), and chronic ( $>60$ days) according to the time interval from symptom onset as described in previously published studies. ${ }^{3,10}$ Patient baseline information including age, sex, comorbidities (hypertension, diabetes, hyperlipidemia), smoking, and stages of VBDAs were recorded.

\section{HRMRI and Vessel Wall Imaging Assessment}

High-resolution MR images were obtained with two 3-T MRI machines using 8-channel head coils at each site. The scan parameters are shown in Table 1. T1- and T2-weighted images were obtained using fast spin-echo (FSE) sequences (Signa TwinSpeed, GE Healthcare) or turbo spin-echo (TSE) sequences (Magnetom Trio [Tim System], Siemens Healthcare). For each site, 3D time-of- flight (3D-TOF) imaging was performed first to locate the aneurysm. Fat- and blood-suppressed, thin-slice T2- and T1-weighted images covering the site of each lesion were then obtained. Fat-saturation technique was applied for all sequences except for 3D-TOF imaging. Quartic or double inversion recovery sequences were used in T1-weighted imaging. Axial plane images were acquired for all postcontrast T1-weighted images 5 minutes after Gd injection $(0.1 \mathrm{mmol} / \mathrm{kg}$ gadopentetate dimeglumine, Magnevist; Bayer Schering Pharma AG) using parameters identical to those of the precontrast T1-weighted images.

Two experienced radiologists (each with 10 years of experience in vascular neuroimaging) blinded to the clinical data independently reviewed the images. The VBDA was first identified on the TOF image. Two-dimensional shortaxis images of the aneurysm generated from pre- and postcontrast T1-weighted images were used to identify wall enhancement. The wall enhancement was defined as complete enhancement when the entire aneurysm wall was enhanced after contrast agent infusion; otherwise, it was defined as partial enhancement when only part of the aneurysm wall was enhanced. The degree of enhancement was assessed based on a visual grading system as follows: Grade 0, not enhanced; Grade 1, similar to that of normal vessel walls but less than that of muscle; and Grade 2, similar to or greater than that of muscle. ${ }^{10}$ Disagreements were resolved by a third radiologist with 15 years of experience in vascular neuroimaging. The maximum diameter of each VBDA at the level of the lesion's largest diameter was measured on HRMRI, including both the dilated aneurysm and the IMH if present.

\section{General Protocol for Reconstructive EVT}

At our institute, patients with unruptured VBDAs with an obvious mass effect are treated as soon as possible if they can tolerate EVT. The first-choice operation for these patients is internal trapping if collateral blood supply is sufficient, while stent-assisted coil embolization or sole stenting is a viable alternative. However, patients with unruptured VBDAs and no obvious mass effect were initially evaluated with MRI/MRA and treated conservatively. If the dissection site was shown to be continuously enlarged on follow-up MRI or MRA, or if the symptoms (e.g., ischemic events) recurred, angiography was performed to further evaluate the lesion. Reconstructive or deconstructive EVT was then scheduled after a risk/benefit evaluation for selected patients. The stent-assisted coiling reconstructive technique was selected for lesions with an apparent dilation requiring coil embolization, while stenting alone was selected for lesions without enough space for coil embolization after stent placement.

Endovascular procedures were performed while patients were under general anesthesia. Patients were premedicated with a dual antiplatelet regimen $(75 \mathrm{mg}$ of clopidogrel and $100 \mathrm{mg}$ of aspirin daily) for 5 days before the operation. Both antiplatelet agents were given orally once daily for 6 weeks after the procedure. Following this treatment, $100 \mathrm{mg}$ of aspirin daily was continued for the next 6 months. Platinum coils were used to embolize the aneurysms, and self-expanding neurovascular stents were used to reconstruct the entire dissected segment. 
TABLE 1. Parameters of the two 3-T MR machines used in this study

\begin{tabular}{lcccc}
\hline \multicolumn{1}{c}{ Parameters } & 3D-TOF & T1 QIR/DIR & T1+C QIR/DIR & T2 FSE/SPACE \\
\hline Repetition time $(\mathrm{msec})$ & $21(22)$ & $800(750)$ & $800(750)$ & $3,000(3020)$ \\
\hline Echo time $(\mathrm{msec})$ & $3.2(3.86)$ & $8.6(12)$ & $8.6(12)$ & $62.3(50)$ \\
\hline Field of view $(\mathrm{cm})$ & $16 \times 16$ & $16 \times 16$ & $16 \times 16$ & 2 \\
\hline Matrix & $256 \times 256$ & $256 \times 320(320 \times 320)$ & $256 \times 320(320 \times 320)$ & $256 \times 320(256 \times 256)$ \\
\hline Thickness $(\mathrm{mm})$ & $1(0.9)$ & 2 & 2 & 2 \\
\hline $\begin{array}{l}\text { DIR = double inversion recovery; QIR = quartic inversion recovery; SPACE = Sampling Perfection with Application-optimized Contrasts using } \\
\text { different flip-angle Evolutions; T1 + C = T1-weighted imaging with contrast. }\end{array}$ \\
FSE and TSE are different sequences used for the two 3-T MR machines. The parameters for the TSE sequences are noted in parentheses.
\end{tabular}

The various embolic materials used during EVT included detachable coils such as the Matrix coil (Cordis) and Microplex coil (MicroVention). Self-expanding neurovascular stents such as the Enterprise stent (Cordis Neurovascular), Solitaire AB stent (ev3), and Low-Profile Visualized Intraluminal Support Device (MicroVention) were used to reconstruct the dissected artery. The metal coverage of the Solitaire and Enterprise stents is 7.22\% and $11.89 \%$ in 3 -mm vessels and $5.42 \%$ and $8.92 \%$ in 4-mm vessels, respectively. ${ }^{8}$ The average metal coverage of the Low-Profile Visualized Intraluminal Support stent is approximately $23.00 \%$ in 3-4-mm vessels according to its product description.

\section{Angiography Assessment}

The DSA findings were reviewed by 2 experienced interventional neuroradiologists, each with 10 years of experience in interventional neuroradiology, and disagreements were resolved by a third radiologist with 15 years of experience in interventional neuroradiology. EVT details including treatment modality, immediate obliteration grade, type of stents used, and important branches involved were recorded, as were angiographic follow-up outcomes. The immediate obliteration grade was defined as complete obliteration if the aneurysmal sac was not visualized after the endovascular procedure, and partial obliteration if contrast agent was still present in the aneurysmal sac. The important vessel branches were the posterior inferior cerebellar artery, anterior inferior cerebellar artery, superior cerebellar artery, posterior cerebral artery, anterior spinal artery, or other large perforating arteries.

\section{Radiological Follow-Up}

Lifelong radiological follow-up was recommended for patients in this study. At approximately 6 months, followup DSA was performed to observe the reconstruction outcome of the vessel lumen and conventional HRMRI (T1weighted imaging, T2-weighted imaging, and 3D-TOF MRA) was performed mainly to observe the change in the size of the lesions after treatment. However, enhanced T1-weighted imaging was not routinely recommended for follow-up MRI because the signal of the artery wall (especially for lesions without an obvious IMH) was susceptible to metal artifacts associated with the stents and coils after EVT. If the lesion was confirmed to be stable on DSA, the patient was then followed up annually with conventional HRMRI. If the lesion was confirmed to be recurrent on DSA and a second EVT was performed, then follow-up angiographic examination was performed using DSA and conventional HRMRI approximately 6 months after the second treatment. Until the lesion was confirmed to be stable on DSA, the patient was followed up annually by conventional HRMRI. The last follow-up time (for stable cases) and the follow-up period during which lesion progression was first confirmed (for progression cases) were recorded and set as the end point in the Cox regression.

Aneurysm progression was defined as either aneurysm recurrence on DSA or enlargement of the external diameter of an aneurysm; otherwise, the lesion was considered stable. Angiographic recurrence was defined as a substantial increase in the contrast medium-filled portion of the dissecting aneurysm compared with a control angiogram taken immediately after the initial treatment. ${ }^{7}$ The 3 maximum external diameters of each VBDA on axial, coronal, and sagittal MR images at the level of the lesion's largest diameter were measured on both pretreatment and follow-up MR images. The external diameter of a VBDA included both the dilated aneurysm and the IMH if present. External diameter enlargement was defined as either $\geq 2.0$ - $\mathrm{mm}$ growth in at least 1 direction among the maximum axial, coronal, and sagittal diameters or $\geq 1.0-\mathrm{mm}$ growth in 2 directions among the maximum axial, coronal, and sagittal diameters.

\section{Statistical Analysis}

Patient age and the maximum diameter of the dissecting aneurysm are presented as means and standard deviations. The 1-sample Kolmogorov-Smirnov test was used to identify normally distributed variables. One-way ANOVA or the Mann-Whitney U-test was used to investigate the relationship between wall enhancement and potential risk factors. Univariate analysis of factors, including the characteristics of the patients and aneurysms, was performed to determine the association of aneurysm progression with other potential risk factors. Variables with a p value $<0.05$ in the univariate analysis were candidates for inclusion in the multivariate Cox proportional hazard regression. All data processing and statistical analyses were performed using SPSS (version 22.0, IBM Corp.). Statistical significance was defined as $\mathrm{p}<0.05$. 


\section{Results}

In total, 82 patients (12 women and 70 men) with 83 aneurysms were enrolled in this study; 1 patient had 2 aneurysms. The mean age of the patients was $53.48 \pm 9.23$ years. The mean maximum diameter of the VBDAs was $11.30 \pm 7.90 \mathrm{~mm}$. Wall enhancement (Grade 1 and Grade 2) was observed in 43 VBDAs $(51.80 \%)$ in this study (Table 2). The wall enhancement was partial in 33 VBDAs (76.74\%) and complete in $10(23.36 \%)$. An IMH was present in 48 VBDAs (57.83\%; Table 3). One significant characteristic of the VBDAs with a larger IMH was the occurrence of subacute bleeding in the circumferential part of the IMH, which is not connected to the lumen and was rarely enhanced, while the intimal flap and the IMH close to the lumen were usually enhanced. The stent-assisted coiling reconstructive technique was used in 62 VBDAs (74.70\%) and sole stenting in 21 VBDAs $(25.30 \%)$. Angiographic follow-up was performed for a mean of 10.55 months (range 6-45 months). Aneurysm progression was observed in 15 VBDAs (18.07\%) and stability in 68 VBDAs (81.93\%; Table 3).

\section{Relationship Between Wall Enhancement and Potential Influencing Factors}

According to the degree of VBDA wall enhancement, the patients were allocated to either the enhancement group (Grades 1 and 2) or no-enhancement group (Grade 0 ). The aneurysm size was $28.06 \%$ larger in the enhancement group than in the no-enhancement group, although the difference did not reach statistical significance $(\mathrm{p}=$ 0.11). The Mann-Whitney U-test also showed no significant differences in age, sex, risk factors, or VBDA stages (the period from onset to MRI performance) between the 2 groups (Table 2 ).

\section{Results of Radiographic Follow-Up and Risk Factors for Aneurysm Progression}

The aneurysm progression and risk factors among all 83 VBDAs are shown in Table 3. Univariate Cox analysis showed that both maximum diameter of the VBDAs and wall enhancement were associated with recurrence ( $p$ $<0.05$ ). Although the rates of complete obliteration and metal coverage were higher in the stable group than in the progression group (28\% vs $20 \%$, and $20.88 \%$ vs $20.72 \%$, respectively), neither of them could predict lesion progression according to univariate analysis $(p>0.05)$. None of the other factors could predict lesion progression according to univariate analysis either $(\mathrm{p}>0.05)$. Multivariate Cox analysis showed that both maximum diameter of the VBDA and wall enhancement were independent risk factors for aneurysm progression $(\mathrm{p}<0.05)$. VBDAs with a small maximum diameter had a lower rate of progression (odds ratio [OR] 1.05, 95\% confidence interval [CI] 1.001.10). Compared with VBDAs with enhancement Grade 0 , VBDAs with enhancement Grade 1 had a significantly higher rate of lesion progression (OR 8.96, 95\% CI 1.0378.25; $\mathrm{p}=0.04$ ); VBDAs with enhancement Grade 2 also had a significantly higher rate of lesion progression (OR 9.90, 95\% CI 1.19-82.22; $\mathrm{p}=0.03$ ).

Eight patients also received enhanced HRMRI follow-
TABLE 2. Relationships between wall enhancement and potential risk factors

\begin{tabular}{lccc}
\hline \multicolumn{1}{c}{ Variable } & $\begin{array}{c}\text { Wall Unenhanced } \\
(\mathrm{n}=40)\end{array}$ & $\begin{array}{c}\text { Wall Enhanced } \\
(\mathrm{n}=43)\end{array}$ & $\begin{array}{c}\mathrm{p} \\
\text { Value }\end{array}$ \\
\hline Mean age $\pm \mathrm{SD}(\mathrm{yrs})$ & $54.45 \pm 8.66$ & $52.58 \pm 9.65$ & 0.36 \\
\hline Sex $(\%)$ & & & 0.63 \\
\hline Male & $35(87.50)$ & $36(83.72)$ & \\
\hline Female & $5(12.50)$ & $7(16.28)$ & \\
\hline Risk factors* $(\%)$ & & & 0.79 \\
\hline$\geq 2$ & $16(40.00)$ & $16(37.21)$ & \\
\hline$<2$ & $24(60.00)$ & $27(62.79)$ & \\
\hline Mean max diameter & $9.87 \pm 6.68$ & $12.64 \pm 8.83$ & 0.11 \\
\pm SD (mm) & & & \\
\hline Onset period $(\%)$ & & & 0.26 \\
\hline Acute & $2(5.00)$ & $0(0)$ & \\
\hline Early subacute & $8(20.00)$ & $8(18.60)$ & \\
\hline Late subacute & $15(37.50)$ & $15(34.88)$ & \\
\hline Chronic & $15(37.50)$ & $20(46.51)$ & \\
\hline
\end{tabular}

* Risk factors included high blood pressure, smoking, diabetes mellitus, and a high cholesterol level.

up (4 progression cases and 4 stable cases). The 4 patients with progression, whose aneurysms were all recurrent on DSA, all showed an enlargement of the external diameter on follow-up HRMRI. Both the pretreatment and followup HRMRI of the 4 patients with progression showed wall enhancement (Grade 1 or Grade 2). The 4 stable patients, whose aneurysms were confirmed to be completely occluded on follow-up DSA, had no change in size of the lesions on follow-up HRMRI. Both the pretreatment and follow-up HRMRI of the 4 stable patients showed no wall enhancement (Grade 0) or mild wall enhancement (Grade 1).

\section{Discussion}

In the present study, aneurysm wall enhancement on HRMRI before EVT and a larger aneurysm size were more frequently observed in progressive (e.g., Figs. 1-3) than stable VBDAs (e.g., Fig. 4) after reconstructive EVT.

Many studies have investigated the risk factors for recurrence of VBDAs after reconstructive EVT., ${ }^{1,16}$ These reported risk factors include aneurysm size, patient age, stent number, and immediate obliteration grade of the aneurysm sac. ${ }^{17,16}$ However, few of these studies included the stability of the arterial wall as a risk factor. VBDA is more likely to represent a disease of the vessel wall than a disease of the vessel lumen. ${ }^{2}$ Instability of the vessel wall (e.g., due to inflammation) may play an important role in the recurrence of VBDAs after reconstructive EVT. Therefore, we included wall enhancement as an indicator and found that wall enhancement and aneurysm size were independent predictors of a high aneurysm progression rate.

Many observations indicate that aneurysm wall enhancement can be used as an indirect marker of vessel wall inflammation and therefore as a potential marker of 
TABLE 3. Multivariate Cox regression analysis for factors associated with progressive VBDA status

\begin{tabular}{|c|c|c|c|c|c|c|}
\hline \multirow[b]{2}{*}{ Variable } & \multirow{2}{*}{$\begin{array}{l}\text { Progressive } \\
\quad(n=15)\end{array}$} & \multirow{2}{*}{$\begin{array}{c}\text { Stable } \\
(n=68)\end{array}$} & \multirow{2}{*}{$\begin{array}{l}\text { Univariate } \\
\text { p Value }\end{array}$} & \multicolumn{3}{|c|}{ Multivariate Cox Regression } \\
\hline & & & & $p$ Value & OR & $95 \% \mathrm{Cl}$ \\
\hline Mean age \pm SD (yrs) & $55.07 \pm 8.87$ & $53.13 \pm 9.28$ & 0.38 & - & - & - \\
\hline $\operatorname{Sex}(\%)$ & & & 0.59 & - & - & - \\
\hline Male & $14(93.33)$ & $57(83.82)$ & - & - & - & - \\
\hline Female & $1(6.67)$ & $11(16.18)$ & - & - & - & - \\
\hline Mean max diameter $\pm \mathrm{SD}(\mathrm{mm})$ & $19.72 \pm 10.71$ & $9.45 \pm 5.63$ & $<0.01$ & 0.04 & 1.05 & $1.00-1.10$ \\
\hline $\mathrm{IMH}(\%)$ & & & 0.06 & - & - & - \\
\hline Yes & $13(86.67)$ & $35(51.47)$ & - & - & - & - \\
\hline No & $2(13.33)$ & $33(48.53)$ & - & - & - & - \\
\hline Mean metal coverage \pm SD (\%) & $20.72 \pm 7.55$ & $20.88 \pm 14.23$ & 0.67 & - & - & - \\
\hline Risk factors $(\%)^{*}$ & & & 0.67 & - & - & - \\
\hline$\geq 2$ & $8(53.33)$ & $24(35.29)$ & - & - & - & - \\
\hline$<2$ & $7(46.67)$ & $44(64.71)$ & - & - & - & - \\
\hline Important branches involved (\%) & & & 0.45 & - & - & - \\
\hline Yes & $2(13.33)$ & $17(25.00)$ & - & - & - & - \\
\hline No & $13(86.67)$ & $51(75.00)$ & - & - & - & - \\
\hline Treatment modality (\%) & & & 0.06 & - & - & - \\
\hline Stent-assisted coiling & $8(53.33)$ & $54(79.41)$ & - & - & - & - \\
\hline Stent only & $7(46.67)$ & $14(20.59)$ & - & - & - & - \\
\hline Wall enhancement (\%) & & & 0.04 & - & - & - \\
\hline Grade 0 & $1(6.67)$ & $39(57.35)$ & - & - & Ref & - \\
\hline Grade 1 & $5(33.33)$ & $18(26.47)$ & - & 0.04 & 8.96 & $1.03-78.25$ \\
\hline Grade 2 & $9(60.00)$ & $11(16.18)$ & - & 0.03 & 9.90 & $1.19-82.22$ \\
\hline Immediate obliteration grade (\%) & & & 0.69 & - & - & - \\
\hline Complete & $3(20.00)$ & $19(27.94)$ & - & - & - & - \\
\hline Partial & $12(80.00)$ & $49(72.06)$ & - & - & - & - \\
\hline
\end{tabular}

Ref $=$ reference.

* Risk factors included high blood pressure, smoking, diabetes mellitus, and a high cholesterol level.

aneurysm instability. ${ }^{5,9} \mathrm{Hu}$ et al. ${ }^{5}$ found evidence of inflammation of the aneurysm wall through pathological studies and proved that aneurysm wall enhancement on HRMRI is highly consistent with pathological changes of inflammation. According to a report by Iihara et al. ${ }^{6}$ regarding resection of a VBDA with a huge IMH, pathological evidence of inflammatory cell infiltration was present in the IMH.

The detailed mechanism of wall enhancement and the factors that influence this phenomenon remain unknown. The aneurysm size was larger in the enhancement group than in the no-enhancement group in the present study, although this difference did not reach statistical significance. According to a study of cervical artery dissection by Habs and colleagues, ${ }^{3}$ the signal intensity of the dissecting wall varies over time; however, our study proves that there is no significant relationship between the signal intensity of the dissecting wall and the period from onset to MRI performance. One possible explanation for this difference is that our study included a limited number of samples and most of patients had a late subacute or chronic stage VBDA.

The results of this study may be useful for clinical prac- tice. First, enhancement HRMRI, which is noninvasive, reproducible, and assessable by the naked eye without postprocessing, is an easy means of predicting the prognosis of VBDAs before treatment. In VBDAs equally suitable for observation or EVT, wall enhancement on HRMRI may guide management toward more prompt intervention. Second, our findings can remind interventional neuroradiologists to pay close attention to VBDAs with wall enhancement and take prompt action to embolize the aneurysm densely and improve the stent metal coverage in the aneurysm neck, therefore improving the efficacy of EVT. Using the case in Figs. 1-3 as an example, valuable information can be obtained from retrospective analysis of the patient's HRMRI findings before EVT and the DSA findings at the time of the first EVT. The first site of recurrence (Fig. 3A), where the intimal flap and IMH near the lumen were obviously enhanced on HRMRI, was embolized with only loose coils. At the second site of recurrence (Fig. 3A), where the vessel wall near IMH was also enhanced on HRMRI, the 3 Enterprise stents used in the first operation may not have been long enough to cover the whole dissecting artery. These may be the reasons for the repeated recurrences. 


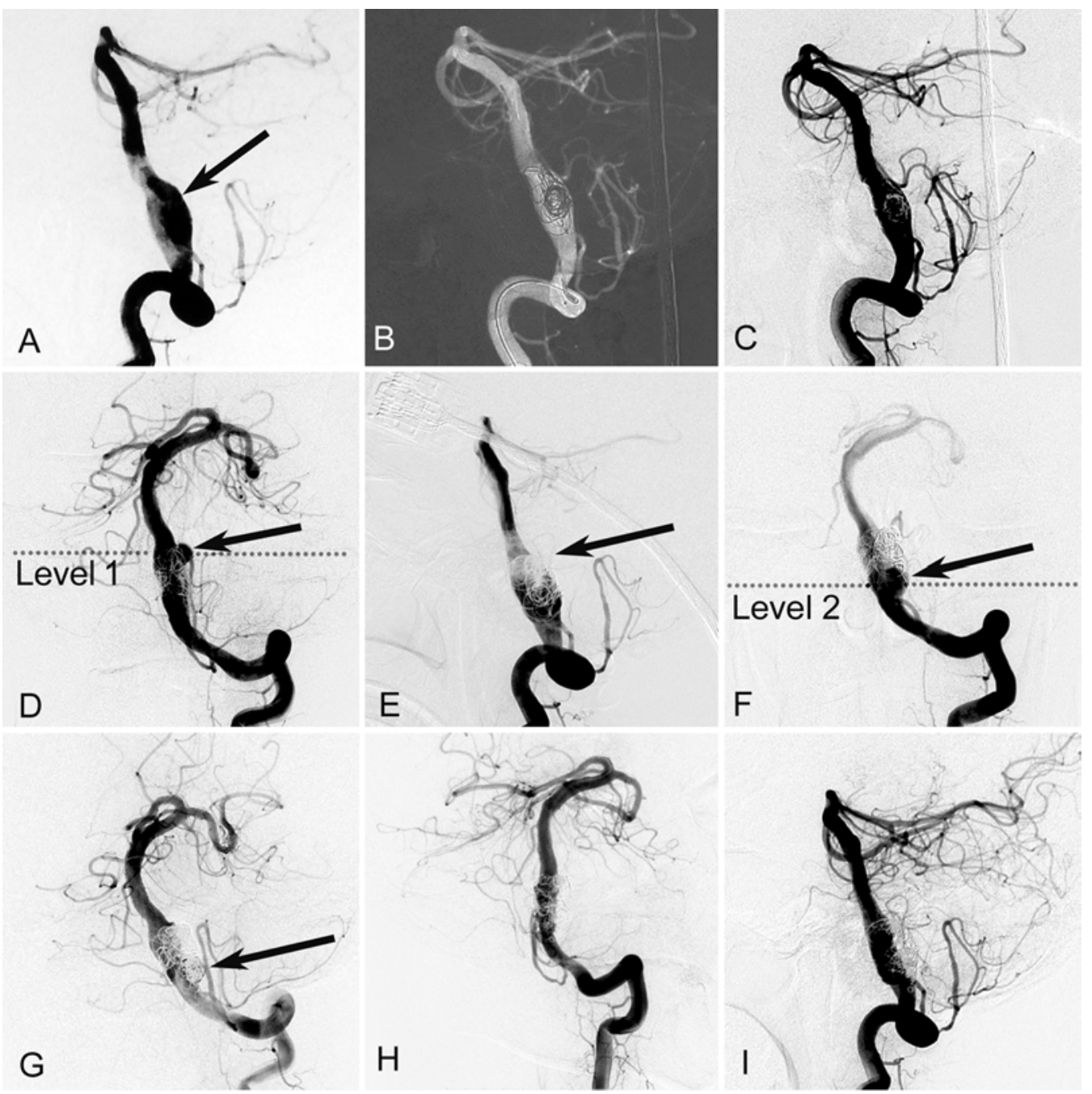

FIG. 1. Recurrent dissecting aneurysm after stent-assisted coil embolization. A: Left vertebral angiogram shows the dissecting aneurysm (arrow) of the vertebrobasilar junction. B: Three Enterprise stents and coils used for the embolization. C: Immediate postprocedural left vertebral angiogram shows the aneurysm was partially embolized. D: Left vertebral angiogram 6 months after stent-assisted coil embolization shows recanalization of the dissecting aneurysm (arrow) at the distal part (Level 1). E: The second treatment was performed with another Enterprise stent placement and coil occlusion (arrow). F: Left vertebral angiogram 12 months after the first EVT shows recanalization of the dissecting aneurysm (arrow) at the proximal part (Level 2). G: The third treatment was performed with 2 Low-Profile Visualized Intraluminal Support Device stents and coil occlusion (arrow). H and I: Left vertebral angiograms 18 months after the first intervention show complete embolization of the dissecting aneurysm.

The aneurysm size was also found to be an independent predictor of aneurysm progression, with larger aneurysms associated with a higher progression rate. This finding has been reported by many authors. ${ }^{16}$ Large VBDAs, which are believed to develop from small, unstable VBDAs, might be even less stable than small VBDAs. Additionally, large VBDAs generally have a longer dissecting segment and aneurysm neck, making them difficult to completely occlude. These might be the reasons for the association between larger aneurysms and higher progression rates after reconstructive EVT.

The obliteration grade of the aneurysm and metal coverage of the stent in the aneurysm neck were believed to be important factors for a low aneurysm recurrence rate after reconstructive EVT. ${ }^{16}$ In this study, the rates of both complete obliteration and metal coverage were higher in the stable group than that in the progression group, although the difference was not statistically significant. However, we still think the 2 factors were important for aneurysm recurrence after reconstructive EVT, and we always strive to achieve dense embolism of the aneurysm and high metal coverage of the stent in the aneurysm neck (e.g., overlapping stents) in our clinical practice. The limited number of cases from a single institution might explain why these 2 factors were excluded from the risk factors for aneurysm progression in the multivariate Cox proportional hazard regression analysis.

\section{Limitations to the Study}

There are some limitations to this study, such as patient selection bias and limited number of cases from a single 


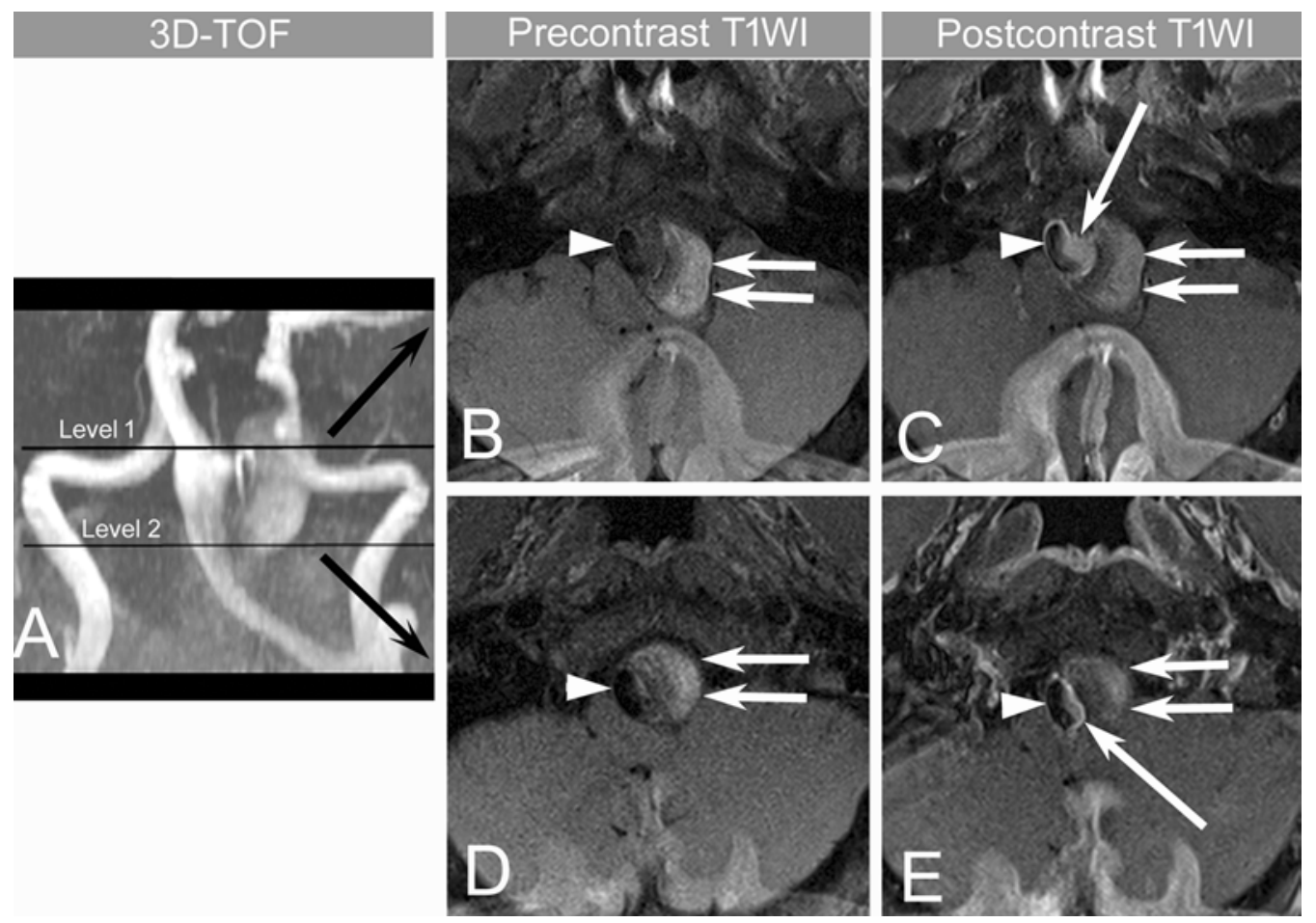

FIG. 2. HRMRI of the patient in Fig. 1 performed before EVT. A: 3D-TOF shows the dissecting aneurysm with a giant IMH. Level 1 and Level 2 are positioned by the twice aneurysm recanalization site. The arrows indicate that panels $B$ and $C$ are MRI slices from Level 1, and panels D and E are MRI slices from Level 2. B and C: Axial precontrast and postcontrast T1-weighted imaging at Level 1 shows the arterial wall (arrowhead) and IMH (single arrow) near the true lumen was enhanced while the circumferential part of the IMH had no enhancement (double arrows). D and E: Axial precontrast and postcontrast T1-weighted imaging at Level 2 shows the wall (arrowhead) near the IMH was enhanced (single arrow) while the circumferential part of the IMH had no enhancement (double arrows).

institution. The follow-up period may be not long enough for some cases and a longer follow-up period was needed to verify the conclusion. The enhancement grading system based on observation only in this study may not be sufficiently objective to avoid artificial error. The progression of VBDAs after reconstructive EVT is a complex process that can be influenced by many factors, but the Cox regression model in this study cannot include all risk factors. The aneurysms were heterogeneous (e.g., differences in treatment modality, follow-up interval, embolic material, aneurysm configuration, and potentially underlying pathology) and these may be confounding factors in this study. Additionally, flow diverter stents were not used in this study because the Pipeline embolization device (ev3) became available in our institute in October 2015, and we also began treating VBDAs with this device after obtaining IRB approval in July 2016. The cases treated by the Pipeline embolization device were not included in this study because of the limited number of patients and lack of angiographic follow-up. A further study is needed to evaluate patients who have been treated with flow-diverting devices to verify the conclusions in the present study.

\section{Conclusions}

Wall enhancement on HRMRI might predict an unsteady state of VBDAs. Aneurysm size and wall enhance-

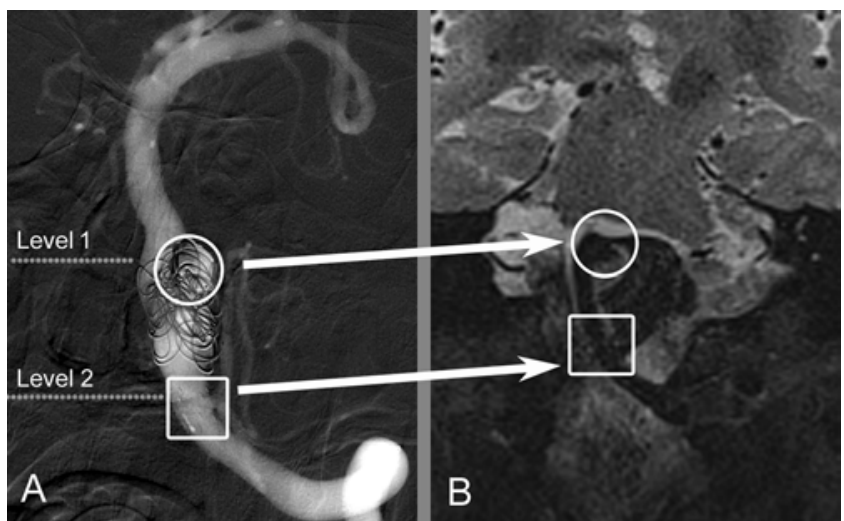

FIG. 3. The causal analysis for aneurysm recurrences on DSA and HRMRI. The patient represented by these images is the same patient as in Figs. 1 and 2. Anteroposterior view of the left vertebral angiogram (A) on DSA after the first EVT and the coronary T2-weighted HRMRI image (B) before EVT of the same patient in Figs. 2 and 3. The first recanalization site was embolized using only loose coils (circle) and the second recanalization site was not well covered by stents (square; proximal stent markers can be seen), which may be the potential reasons for the 2 recanalizations. 

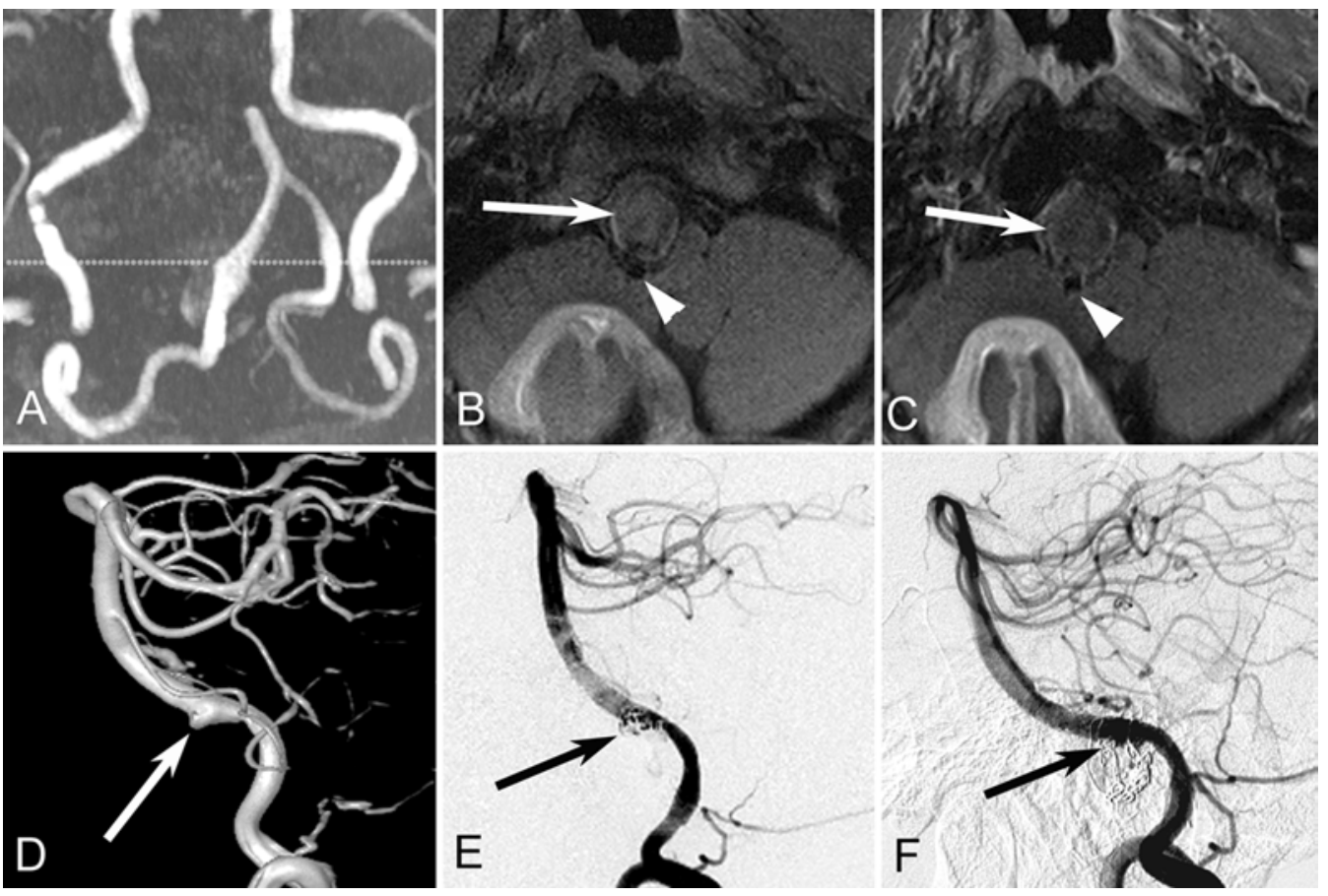

FIG. 4. HRMRI and DSA in a stable aneurysm with no wall enhancement. A: 3D-TOF shows the dissecting aneurysm with a large IMH. B and C: Axial precontrast (B) and postcontrast (C) T1-weighted imaging shows the arterial wall (arrowhead) and IMH (arrow) were not enhanced. D: Right vertebral angiogram shows the dissecting aneurysm (arrow) of the vertebral artery. E: Immediate postprocedural right vertebral angiogram shows the aneurysm was completely embolized by stent-assisted coil embolization (arrow). F: Right vertebral angiogram 32 months after stent-assisted coil embolization shows perfect reconstruction of the dissecting artery (arrow) without aneurysm recanalization.

ment on HRMRI can predict the progression of VBDAs after reconstructive EVT. These findings can remind interventional neuroradiologists to pay close attention to large VBDAs with wall enhancement on MRI and take prompt action in endovascular procedures, in turn improving the efficacy of EVT.

\section{Acknowledgments}

This work was supported by the National Natural Science Foundation of China (grant nos. 81301003, 81301193, 81371315, 81471167, and 81220108007), Beijing Natural Science Foundation (grant no. 7126056), and Special Research Project for Capital Health Development (grant no. 2014-1-1071).

\section{References}

1. Crobeddu E, Lanzino G, Kallmes DF, Cloft HJ: Review of 2 decades of aneurysm-recurrence literature, part 1: reducing recurrence after endovascular coiling. AJNR Am J Neuroradiol 34:266-270, 2013

2. Debette S, Compter A, Labeyrie MA, Uyttenboogaart M, Metso TM, Majersik JJ, et al: Epidemiology, pathophysiology, diagnosis, and management of intracranial artery dissection. Lancet Neurol 14:640-654, 2015

3. Habs M, Pfefferkorn T, Cyran CC, Grimm J, Rominger A, Hacker M, et al: Age determination of vessel wall hematoma in spontaneous cervical artery dissection: a multi-sequence 3T cardiovascular magnetic resonance study. J Cardiovasc Magn Reson 13:76, 2011

4. Horie N, Morikawa M, Fukuda S, Hayashi K, Suyama K, Nagata I: Detection of blood blister-like aneurysm and intramural hematoma with high-resolution magnetic resonance imaging. J Neurosurg 115:1206-1209, 2011

5. Hu P, Yang Q, Wang DD, Guan SC, Zhang HQ: Wall enhancement on high-resolution magnetic resonance imaging may predict an unsteady state of an intracranial saccular aneurysm. Neuroradiology 58:979-985, 2016

6. Iihara K, Murao K, Sakai N, Soeda A, Ishibashi-Ueda $\mathrm{H}$, Yutani C, et al: Continued growth of and increased symptoms from a thrombosed giant aneurysm of the vertebral artery after complete endovascular occlusion and trapping: the role of vasa vasorum. Case report. J Neurosurg 98:407-413, 2003

7. Kim BM, Shin YS, Kim SH, Suh SH, Ihn YK, Kim DI, et al: Incidence and risk factors of recurrence after endovascular treatment of intracranial vertebrobasilar dissecting aneurysms. Stroke 42:2425-2430, 2011

8. Krischek O, Miloslavski E, Fischer S, Shrivastava S, Henkes $\mathrm{H}$ : A comparison of functional and physical properties of self-expanding intracranial stents [Neuroform3, Wingspan, Solitaire, Leo+, Enterprise]. Minim Invasive Neurosurg 54:21-28, 2011

9. Nagahata S, Nagahata M, Obara M, Kondo R, Minagawa N, Sato $S$, et al: Wall enhancement of the intracranial aneurysms revealed by magnetic resonance vessel wall imaging using three-dimensional turbo spin-echo sequence with motionsensitized driven-equilibrium: a sign of ruptured aneurysm? Clin Neuroradiol 26:277-283, 2016

10. Park KJ, Jung SC, Kim HS, Choi CG, Kim SJ, Lee DH, et al: Multi-contrast high-resolution magnetic resonance findings of spontaneous and unruptured intracranial vertebral artery dissection: qualitative and quantitative analysis according to stages. Cerebrovasc Dis 42:23-31, 2016 
11. Sikkema T, Uyttenboogaart M, Eshghi O, De Keyser J, Brouns R, van Dijk JM, et al: Intracranial artery dissection. Eur J Neurol 21:820-826, 2014

12. Song Y, Wang Y, Li C, Wang Y, Mu S, Yang X: Retreatment and outcomes of recurrent intracranial vertebral artery dissecting aneurysms after stent assisted coiling: a single center experience. PLoS One 9:e113027, 2014

13. Takano K, Yamashita S, Takemoto K, Inoue T, Kuwabara Y, Yoshimitsu K: MRI of intracranial vertebral artery dissection: evaluation of intramural haematoma using a black blood, variable-flip-angle 3D turbo spin-echo sequence. Neuroradiology 55:845-851, 2013

14. Tsukahara T, Minematsu K: Overview of spontaneous cervicocephalic arterial dissection in Japan. Acta Neurochir Suppl 107:35-40, 2010

15. Wang Y, Lou X, Li Y, Sui B, Sun S, Li C, et al: Imaging investigation of intracranial arterial dissecting aneurysms by using 3 T high-resolution MRI and DSA: from the interventional neuroradiologists' view. Acta Neurochir (Wien) 156:515-525, 2014

16. Zhao KJ, Fang YB, Huang QH, Xu Y, Hong B, Li Q, et al: Reconstructive treatment of ruptured intracrania spontaneous vertebral artery dissection aneurysms: long- term results and predictors of unfavorable outcomes. PLoS One 8:e67169, 2013

\section{Disclosures}

The authors report no conflict of interest concerning the materials or methods used in this study or the findings specified in this paper.

\section{Author Contributions}

Conception and design: Yang, Sui. Acquisition of data: Zhang, Tian, Chen. Analysis and interpretation of data: Liu. Drafting the article: Zhang. Critically revising the article: Sui, Wang, Wu. Reviewed submitted version of manuscript: Yang, Sui, Wang, $\mathrm{Wu}$. Approved the final version of the manuscript on behalf of all authors: Yang. Statistical analysis: Liu. Administrative/technical/ material support: Wu. Study supervision: Liu.

\section{Correspondence}

Xinjian Yang, Department of Interventional Neuroradiology, Beijing Neurosurgical Institute and Beijing Tian Tan Hospital, Capital Medical University, Beijing 100050, China. email: yangxinjian@bjttyy.org. 